\title{
Patterns of Mind-Body Therapies in Adults with Common Neurological Conditions
}

\author{
Rebecca Erwin Wells $^{a}$ Russell S. Phillips ${ }^{b}$ Ellen P. McCarthy ${ }^{b}$ \\ a Department of Neurology and ${ }^{b}$ Division of General Medicine and Primary Care, Department of Medicine, \\ Beth Israel Deaconess Medical Center, Harvard Medical School, Boston, Mass., USA
}

\section{Key Words}

Mind-body therapies $\cdot$ Headache $\cdot$ Migraine $\cdot$ Back pain .

Stroke $\cdot$ Dementia $\cdot$ Seizures $\cdot$ Memory impairment .

Epidemiology

\begin{abstract}
Background: Over $40 \%$ of adults with common neurological conditions use complementary and alternative medicine, and mind-body therapies are the most commonly used form. Our objective was to describe mind-body use in adults with common neurological conditions. Methods: We compared mind-body use between adults with and without common neurological conditions (regular headaches, migraines, back pain with sciatica, strokes, dementia, seizures or memory loss) using the 2007 National Health Interview Survey of 23,393 sampled American adults. Results: Adults with common neurological conditions used mind-body therapies more frequently than those without (24.5 vs. $16.6 \%, p<0.0001)$; differences persisted after adjustment. Deep breathing exercises, meditation and yoga were used most frequently. Nearly $70 \%$ of the adults with common neurological conditions did not discuss their mind-body use with their health care provider. Those with neurological con-
\end{abstract}

ditions used mind-body therapies more than those without these conditions because of provider recommendation ( 26 vs. $13 \%$ ) or because conventional treatments were perceived ineffective (12 vs. $4 \%$ ) or too costly (7 vs. $2 \%$ ), respectively. Conclusions: Mind-body therapies are used more frequently among adults with common neurological conditions, more often when conventional treatments were perceived ineffective. More research is warranted on the efficacy of mind-body use for common neurological conditions.

Copyright $\odot 2010$ S. Karger AG, Basel

\section{Introduction}

Mind-body therapies are a form of complementary and alternative medicine (CAM). The National Center for Complementary and Alternative Medicine (NCCAM) defines mind-body medicine as techniques designed to 'enhance the mind's capacity to affect bodily function and symptoms' [1]. These practices often offer stress relief and relaxation [2]. Neurological conditions may be exacerbated by stress [3-5], and thus adults with neurological conditions may turn to stress-relieving mind-body techniques for help. Previously, we analyzed the use of all

\section{KARGER}

(c) 2010 S. Karger AG, Basel

Fax +41613061234

E-Mail karger@karger.ch

www.karger.com
Rebecca Erwin Wells, MD

Department of Neurolog

Beth Israel Deaconess Medical Center, Harvard Medical School

330 Brookline Avenue, Boston, MA 02215 (USA)

Tel. +1 617632 8917, Fax +1 617632 8920, E-Mail rwells@ bidmc.harvard.edu 
forms of CAM among US adults with common neurological conditions using the 2007 National Health Interview Survey (NHIS) and reported that adults with common neurological conditions used CAM more frequently than those without ( 44.1 vs. $32.6 \%, p<0.0001$ ); differences persisted after adjusting for sociodemographics, illness burden, access to care and health habits [6]. Mindbody therapies were the most commonly used CAM therapy by adults with neurological conditions and they had higher rates of mind-body therapy use than the rest of the US population. The age-sex-adjusted prevalence of mindbody use varied across conditions: $22.9 \%$ with seizures and regular headaches used mind-body therapies, $25.4 \%$ with sciatic back pain, $27.5 \%$ with migraines and $27.8 \%$ with memory loss [6]. Because of the importance of mindbody therapy use among adults with common neurological conditions, our objectives for our current analysis are to understand the specific types of mind-body therapies used, patterns of use, reasons for use and correlates of use in adults with common neurological conditions.

\section{Materials and Methods}

\section{Data Source}

The NHIS is an annual nationally representative health survey of the civilian US population that employs a multistage stratified sampling design to select households for face-to-face interviews, conducted in English and/or Spanish [7]. One adult, aged $\geq 18$ years, was randomly selected from each household to answer the Sample Adult questionnaire. In 2007, NHIS administered an alternative medicine supplement to better understand the use of CAM therapies [7]. Participants were asked: 'During the past 12 months, have you used (specific therapy)?' We used data on the use of 10 mind-body therapies listed below. The final adult sample included 23,393 respondents, with an overall response rate of $67.8 \%$ [7].

\section{Common Neurological Conditions}

As NHIS is nationally representative, detailed questions about neurological conditions with a high prevalence in the general population were queried. Of these, we examined the following common neurological conditions specifically: (1) regular headaches within the prior 12 months; (2) memory loss within 12 months; (3) stroke within 12 months; (4) severe headache/migraine within 3 months; (5) low back pain, with pain spreading down either leg below the knee(s) within 3 months; (6) ever having seizures, and (7) ever having dementia.

\section{Outcomes of Interest}

Our primary outcome was use of at least one of the following mind-body therapies within the prior 12 months: biofeedback, energy healing, hypnosis, tai chi, yoga, qi gong, meditation, guided imagery, progressive relaxation or deep breathing exercises. These were the 10 mind-body therapies queried in the NHIS survey and defined as mind-body therapies by NCCAM.
We also examined: (1) disclosure of mind-body use to health care providers, and (2) reason for mind-body use. For each therapy used, the respondents were asked about disclosure to conventional practitioners and reasons for mind-body use. To ascertain reasons for use, the respondents were asked to answer yes/no to each of 7 possible reasons for use (only these 7 options were offered). These included: (1) to improve or enhance energy; (2) for general wellness/general disease prevention; (3) to improve/enhance immune function; (4) because conventional medical treatments did not help; (5) because conventional medical treatments were too expensive; (6) it was recommended by a health care provider, and (7) it was recommended by family, friends or coworkers.

\section{Correlates of Mind-Body Use}

We considered potential correlates of mind-body use reported previously $[8,9]$. Sociodemographic factors included age, sex, race/ethnicity, region of residence, educational attainment, birthplace and marital status. Potential indicators of illness burden included number of ER visits in the prior year, presence of functional limitations, perceived health status, self-reported history of chronic medical conditions (hypertension, hyperlipidemia, diabetes, cancer, coronary artery disease, myocardial infarction) and depression and/or anxiety in the past year. Indicators of access to care included imputed family income provided by NHIS [10], insurance status, delayed care because of worries about cost and delayed care because they could not afford it. Health habit measures included smoking status, physical activity level [11] and alcohol intake [12].

\section{Statistical Analyses}

We used bivariable analyses to compare adults with and without common neurological conditions. We estimated the overall and therapy-specific prevalence of mind-body use, as well as the age-sex-adjusted reasons for and disclosure of mind-body use to healthcare providers. We performed multivariable logistic regression to determine whether differences in the overall prevalence of mind-body use persisted between adults with and without common neurological conditions after adjusting for sociodemographic characteristics, illness burden, access to care and health habits. We used a stepwise backward elimination process, considering factors associated with mind-body use with a p value $<0.15$ in bivariable analyses and those found to be important in other studies $[8,9]$. Factors with a Wald statistic $p$ value of $\leq 0.05$ and conditions that were considered a priori and have been shown to be important in the literature were retained in the final model $[8,9]$. We considered potential confounding by examining a $10 \%$ change in the estimated $\beta$-coefficient for factors that did not meet these criteria. Next, we used logistic regression (as described above) to identify independent correlates of mind-body use in adults with common neurological conditions.

SAS-callable SUDAAN version 9.2 (Research Triangle Park, N.C., USA) was used to account for the complex sampling design, and data were weighted appropriately so that our results reflect national estimates [7]. The study was determined exempt by the institutional review boards at our institutions because of use of de-identified data. 
Table 1. Reasons for use of mind-body therapies, age-sex-adjusted

\begin{tabular}{llc}
\hline Reason & \multicolumn{2}{l}{$\begin{array}{l}\text { Neurological } \\
\text { conditions, \% }\end{array}$} \\
\cline { 2 - 3 } & with & without \\
\hline General wellness/disease prevention & 64 & 69 \\
Improve/enhance energy & 43 & 46 \\
Family/friends recommendation & 31 & 29 \\
Improve/enhance immune function & 21 & 22 \\
Provider recommendation* & 26 & 13 \\
Conventional treatment ineffective* $^{*}$ & 12 & 4 \\
Conventional treatment too expensive* $^{*}$ & 7 & 2 \\
\hline
\end{tabular}

Neurological conditions include regular headaches, migraines, back pain with sciatica, memory loss, seizures, stroke and dementia. Mind-body therapies include biofeedback, energy healing, hypnosis, tai chi, yoga, qi gong, meditation, guided imagery, progressive relaxation and deep breathing exercises.

${ }^{*} \mathrm{p}<0.0001$.

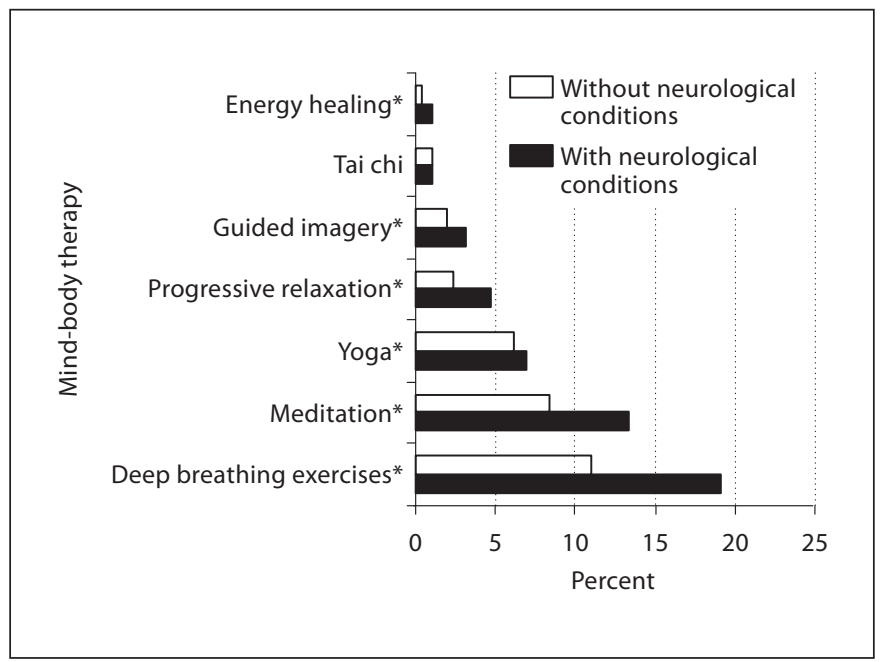

Fig. 1. The prevalence of mind-body therapy use. Biofeedback, hypnosis, and qi gong data not presented because $\mathrm{n}<50$. Neurological conditions include regular headaches, migraines, back pain with sciatica, memory loss, seizures, stroke and dementia. ${ }^{*} \mathrm{p}<0.05$.

\section{Results}

\section{Sample Characteristics}

Overall, 6,587 (28.2\%) sampled adults had at least 1 common neurological condition, representing an estimated 61.6 million Americans nationwide. The respondent characteristics differed significantly between those with and without common neurological conditions, as described previously [6]. Compared to adults without common neurological conditions, those with these conditions were more likely to be women, have lower educational attainment and family incomes, perceive their health as fair or poor, have functional limitations, report delaying care because of worries about cost or because it was not affordable, and report other medical or psychiatric conditions.

\section{Prevalence of Mind-Body Use}

Overall, $24.5 \%$ of the US adults with common neurological conditions reported using at least 1 mind-body therapy within the prior 12 months, representing an estimated 15.1 million adults, as compared to $16.6 \%$ without neurological conditions, $\mathrm{p}<0.0001$. After adjustment for sociodemographic characteristics, illness burden, access to care and health habits, adults with common neurological conditions remained more likely to use mind-body therapies than those without these conditions (adjusted $\mathrm{OR}=1.38,95 \% \mathrm{CI}=1.25-1.53$ ). Of the mind-body therapies, deep breathing exercises, medita-

tion and yoga were most commonly used by those with neurological conditions (19, 13 and $7 \%$, respectively), and those with neurological conditions used these therapies more than those without ( $\mathrm{p}<0.05$ for all comparisons) (fig. 1).

\section{Disclosure of and Reasons for Mind-Body Use}

Sixty-seven percent of the adults with common neurological conditions did not discuss their mind-body use with their health care provider, compared to $76 \%$ of the adults without neurological conditions ( $\mathrm{p}<0.001)$, after adjusting for age and sex. Respondents with neurological conditions used mind-body therapies more often than those without because their provider recommended it $(26$ vs. $13 \%$ ), conventional treatment was ineffective (12 vs. $4 \%$ ), and conventional treatment was too expensive (7 vs. $2 \%)$ ( $p<0.0001$ for all comparisons), after adjusting for age and sex (table 1). The most common reasons for use of mind-body therapies, in both adults with and without common neurological conditions, were general wellness/ disease prevention (64 vs. 69\%), to improve/enhance energy ( 43 vs. $46 \%)$ and family/friends recommendation (31 vs. $29 \%)$, respectively.

\section{Correlates of Mind-Body Use}

Among adults with common neurological conditions, factors significantly associated with mind-body use in- 
Fig. 2. Significant correlates of mind-body use among adults with common neurological conditions. Point estimates reflect adjusted odds ratio (aOR) and bars represent 95\% confidence intervals (CI). Only aOR with $\mathrm{p}<0.001$ and CIs that do not include 0 are presented. Adjusted for sociodemographic characteristics, illness burden, access to care and health habits.

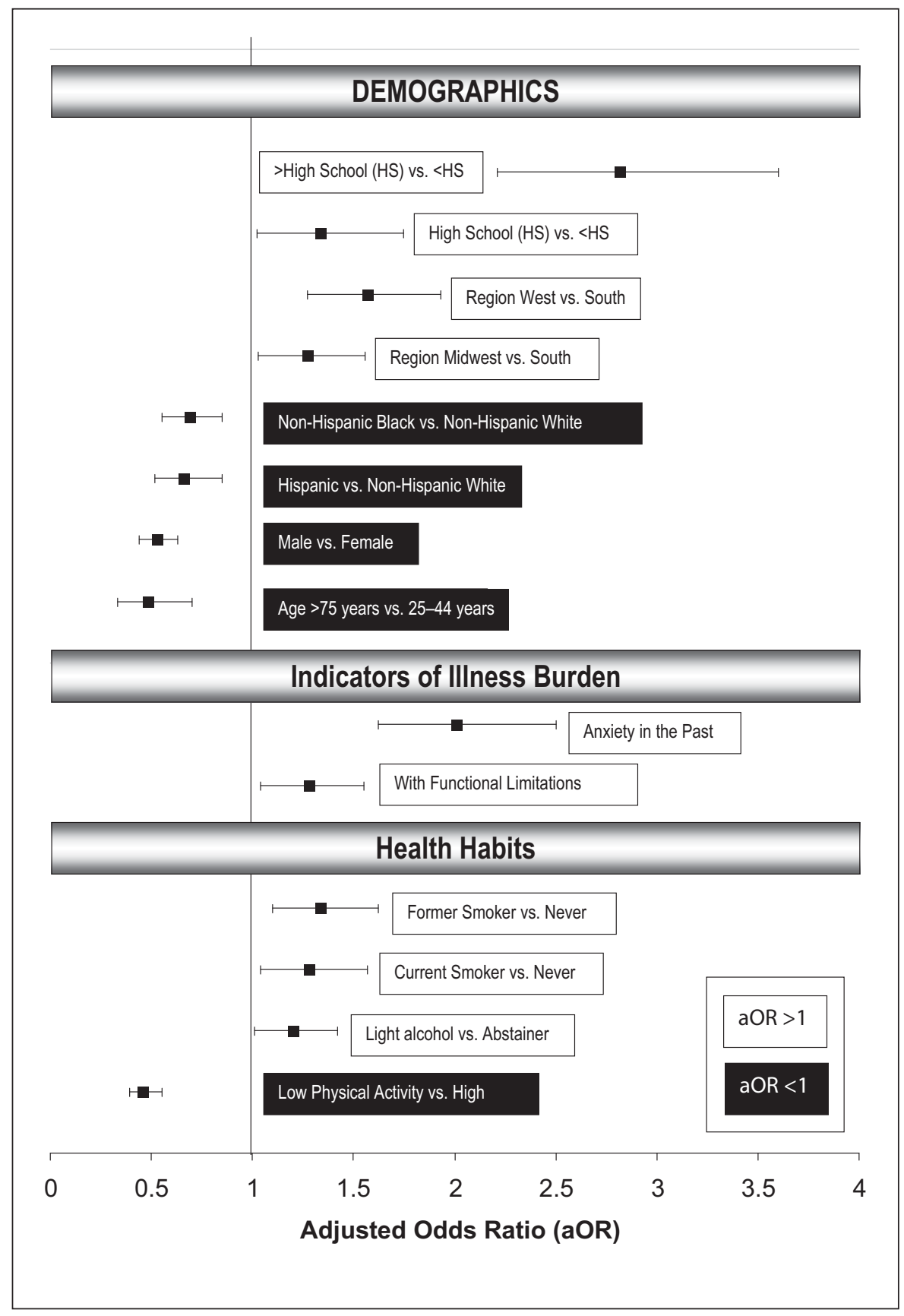

cluded having a high school education or greater, anxiety in the past year, residing in the west or midwest (vs. south) of America, being a current or former smoker, having functional limitations and light alcohol use (fig. 2); factors significantly associated with a lower likelihood of mind-body use included being Non-Hispanic Black or Hispanic (vs. Non-Hispanic White), male, age $>75$ years or low physical activity, after adjustment.

\section{Discussion}

Mind-body therapies were used more frequently among American adults with common neurological conditions than among those without. Deep breathing exercises, meditation and yoga were used most, while biofeedback, hypnosis, and qi gong were used least. The age-sex-adjusted prevalence of mind-body use varied 
across conditions: $22.9 \%$ with seizures and regular headaches used mind-body therapies, $25.4 \%$ with sciatic back pain, $27.5 \%$ with migraines and $27.8 \%$ with memory loss [6]. Despite the high prevalence of mindbody use, $67 \%$ of the adults with common neurological conditions did not discuss their mind-body use with their health care provider. Adults with common neurological conditions were more likely than those without these conditions to report using mind-body therapies because their provider recommended it or because conventional treatments were perceived ineffective or too expensive. Among the adults with neurological conditions, those with more education, anxiety in the past, living in the west or midwest, being a current or former smoker, having functional limitations or with light alcohol use were significantly more likely to report mindbody use. These findings specific to mind-body medicine are consistent with our prior results of all types of CAM use in adults with neurological conditions [6]. This work extends our knowledge from our prior work by focusing on mind-body therapies, which have the potential for treatment of neurological conditions through stress reduction.

Despite the popularity of mind-body therapies among adults with neurological conditions, research on its therapeutic benefits is in its infancy. A recent review evaluating the benefits of mind-body interventions in neurological conditions found that most studies in this area are not high quality, often having small sample sizes and no adequate control groups [13]. The best evidence is for migraines: the US Headache Consortium treatment guidelines suggest that complementary therapies such as relaxation training may be considered as treatment options for prevention of migraine (grade A evidence) [14]. In addition, the American College of Physicians and American Pain Society recommended yoga or progressive relaxation as treatment for refractory chronic/subacute low back pain [15]. There is minimal evidence to support the use of mind-body therapies for memory loss, even though our results showed that this group had the highest use of mind-body therapies.

Further research is needed to continue to evaluate the efficacy of mind-body therapies in these and other neurological conditions. Clinicians should make a concerted effort to ask patients about their mind-body use, especially since many use mind-body therapies because they feel conventional treatments are ineffective or too expensive (nearly 20\%) and thus may be nonadherent to traditional interventions. Furthermore, providers need to know what their patients are using and why, not only to assess adherence to traditional interventions but to grasp an understanding of the impact of the illness on the patient's life and to know what therapies a patient is willing to try. Further, the provider can counsel the patient on the evidence for mind-body therapies for their condition and monitor for any untoward side effects.

Our data revealed that a quarter of the adults with common neurological conditions reported using mindbody therapies because of provider recommendation. Although this is consistent with studies of other conditions $[9,16,17]$, it is interesting because the common perception is that most practitioners only recommend 'evidence-based medicine' and there is actually limited evidence to support the use of mind-body therapies in adults with neurological conditions. Perhaps more providers are recommending these therapies than realized because of the lack of $100 \%$ effective 'traditional' therapies to treat neurological conditions, as they are overall safe interventions that may make patients feel better, and because complementary therapies may be more congruent with patients' beliefs and have fewer side effects than traditional treatment options. Further understanding of the motivating factors for why providers are making these recommendations will be important for future research.

Our study has limitations. NHIS is cross-sectional, subject to misclassification and recall bias, and relies on self-reporting. Relying on self-report can lead to misclassification and inaccurate information because often patients label themselves with a condition that has not actually been evaluated and diagnosed by a medical provider. NHIS only includes details of neurological conditions with a high prevalence in the general population; conditions commonly seen by neurologists, such as multiple sclerosis, are not addressed fully. The severity of conditions is not assessed. There may be mind-body therapies that people use that are not included in the 10 mind-body therapies queried in the NHIS, and thus the results may underestimate the true prevalence of mindbody use in the USA. Moreover, the mind-body techniques queried in this study, such as deep breathing exercises, may be open to interpretation. Although respondents reported use of mind-body therapies from the prior 12 months, some conditions are reported if present in the prior 3 months. Because of small sample sizes, our study could not assess whether mind-body therapies were used specifically for the conditions examined. The survey respondents were only offered 7 options for why they chose to use CAM; there may be other reasons and these were not addressed. 


\section{Conclusion}

This project provides insight into the use of mindbody therapies among American adults with common neurological conditions. We found that mind-body therapies are used more frequently in adults with common neurological conditions than in those without, even after adjustment. Most physicians do not know about their patients' mind-body use, thus it is critical to reinforce the importance of clinicians discussing this with their patients. Although the prevalence of mind-body use among adults with common neurological conditions is high nationally, there is only limited evidence for its efficacy.
Thus, a chasm continues to exist between our scientific knowledge of these therapies and their use by patients. Robust trials are critically needed to bridge this gap.

\section{Acknowledgments and Funding}

R.E.W. was supported by an institutional National Research Service Award No. T32AT000051 from NCCAM at the National Institutes of Health (NIH). R.S.P. was supported by a Mid-Career Investigator Award K24AT000589 and E.P.M. was supported by R03AT002236, also from NCCAM. The content is solely the responsibility of the authors and does not necessarily represent the official views of the NCCAM or the NIH. No conflicts of interest.

\section{References}

1 National center for alternative and complementary medicine: 'What is CAM?' http:// nccam.nih.gov/health/whatiscam/.

$\checkmark 2$ Dusek JA, Benson H: Mind-body medicine: a model of the comparative clinical impact of the acute stress and relaxation responses. Minn Med 2009;92:47-50.

-3 Mohr DC, Hart SL, Julian L, Cox D, Pelletier D: Association between stressful life events and exacerbation in multiple sclerosis: a meta-analysis. BMJ 2004;328:731.

$\checkmark 4$ Wilson RS, Evans DA, Bienias JL, Mendes de Leon CF, Schneider JA, Bennett DA: Proneness to psychological distress is associated with risk of Alzheimer's disease. Neurology 2003;61:1479-1485

5 Wober C, Wober-Bingol C: Triggers of migraine and tension-type headache. Handb Clin Neurol 2010;97:161-172.

6 Wells RE, Phillips RS, Schachter SC, McCarthy EP: Complementary and alternative medicine use among US adults with common neurological conditions. J Neurol 2010; 257:1822-1831.
7 CDC national center for health statistics national health interview survey 2007 survey description document. ftp://ftp.cdcgov/pub/ Health_Statistics/NCHS/Dataset_Docu mentation/NHIS/2007/srvydesc.pdf.

$\checkmark 8$ Bertisch SM, Wee CC, Phillips RS, McCarthy EP: Alternative mind-body therapies used by adults with medical conditions. J Psychosom Res 2009;66:511-519.

9 Yeh GY, Davis RB, Phillips RS: Use of complementary therapies in patients with cardiovascular disease. Am J Cardiol 2006;98: 673-680.

10 CDC national center for health statistics national health interview survey 2007 imputed family income. http://www.cdcgov/NCHS/ nhis/2007imputedincome.htm.

11 Kushi LH, Fee RM, Folsom AR, Mink PJ, Anderson KE, Sellers TA: Physical activity and mortality in postmenopausal women. JAMA 1997;277:1287-1292.

12 Health, United States 2007. http://www. cdcgov/nchs/data/hus/hus07.pdf.
13 Wahbeh H, Elsas SM, Oken BS: Mind-body interventions: applications in neurology. Neurology 2008;70:2321-2328.

14 Campbell JK, Penzien DB, Wall EM, US headache consortium: Evidence-based guidelines for migraine headache: behavioral and physical treatments. 2000. http:// www.aan.com

15 Chou R, Huffman LH, American Pain Society, American College of Physicians: Nonpharmacologic therapies for acute and chronic low back pain: a review of the evidence for an American Pain Society/American College of Physicians clinical practice guideline. Ann Intern Med 2007; 147:492504.

16 Birdee GS, Legedza AT, Saper RB, Bertisch SM, Eisenberg DM, Phillips RS: Characteristics of yoga users: results of a national survey. J Gen Intern Med 2008;23:1653-1658.

17 Kanodia AK, Legedza AT, Davis RB, Eisenberg DM, Phillips RS: Perceived benefit of complementary and alternative medicine (CAM) for back pain: a national survey. J Am Board Fam Med 2010;23:354-362. 